\title{
SIGN-CHANGING SOLUTIONS OF $\left(e_{1}, B\right)$-LIMIT INCREASING OPERATOR EQUATION*
}

\author{
XU XIAN \\ Department of Mathematics, Xuzhou Normal University, Xuzhou, Jiangsu, 221116, P. R. China \\ email:xuxian68@163.com
}

(Received 21 April 2010; accepted 21 September 2010; first published online 10 March 2011)

\begin{abstract}
In this paper, by using the fixed point index method first we obtain some existence and multiplicity results for sign-changing solutions of an $\left(e_{1}, B\right)$-limit increasing operator equation. The main results can be applied to many non-linear boundary value problems to obtain the existence and multiplicity results for signchanging solutions. We also give a clear description of locations of these sign-changing solutions through strict lower and upper solutions. As an example, in the last section we obtain some existence and multiplicity results for sign-changing solutions of some Sturm-Liouville differential boundary value problems.
\end{abstract}

2010 Mathematics Subject Classification. 47H07, 47H10.

1. Introduction. As is well known, when studying non-linear problems, better results may be established if the non-linear terms are assumed to have some monotonicity properties. For instance, when studying non-linear differential boundary value problems, if the non-linear terms are increasing and there exists a pair of wellordered upper and lower solutions, then the differential boundary value problems always have maximal and minimal solutions on the ordered interval defined by the well-ordered upper and lower solutions. For another instance, recently some authors obtained the existence results for critical points by combing the lower and upper solutions method with the descending flowing invariant set method; see $[6,27]$ and the references therein. In order to establish these results, the authors of these papers always assume that the non-linear terms satisfy some kinds of monotonicity properties. However, in many cases the non-linear terms may not have any monotonicity properties. In order to overcome this difficulty, in [13] we introduced a new concept of $\left(e_{1}, B\right)$-limit increasing operator and studied the existence of solutions of $\left(e_{1}, B\right)$-limit increasing operator equations under the condition of pairs of paralleled upper and lower solutions.

The sign-changing solutions have attracted much attention in recent years; see [112, 21-30] and the references therein. Generally speaking, there are three approaches to establish the sign-changing solution results. They are critical point theory, the topological degree method and the global bifurcation theory. In our papers [8-11], we established some existence results for sign-changing solutions of some differential boundary value problems via the topological degree method and the global bifurcation theories. Concerning the method of global bifurcation theories, we also refer readers to

*This paper is supported by NSFC10971179, Natural Science Foundation of Jiangsu Education Committee (09KJB110008) and Qing Lan Project. 
Rabinnowitz's classical results $[\mathbf{2 9}, \mathbf{3 0}]$. Since there are explicit geometrical significance, recently the method of critical point theory has been employed extensively to find signchanging solutions. Bartsch and Wang [27] established an abstract critical point theory in the partially ordered Hilbert spaces by virtue of critical groups and studied signchanging solutions of some elliptic boundary value problems. Concerning the method of critical point theory we also refer readers to Refs. [1-7, 21-26, 28]. Moreover, there were some interesting results in Zou's recent monographs [28] concerning signchanging critical point theory. However, we should point out that in many cases this method is invalid because a suitable variational setting can not be found for some nonlinear boundary value problems, e.g. impulsive differential boundary value problems, multi-point differential boundary value problems etc.; nevertheless we may establish existence results for sign-changing solutions for these differential boundary value problems by using the methods of the topological degree or global bifurcation theories.

The main purpose of this paper is to study the sign-changing solutions of $\left(e_{1}, B\right)$ limit increasing operator equations. Some existence and multiplicity results for the signchanging solutions of an equation of $\left(e_{1}, B\right)$-limit increasing operator are established and the locations of these sign-changing solutions are clearly described in terms of the cone structure of the space. The abstract theorems are applied to the SturmLiouville equations to obtain the existence results for sign-changing solutions. Of course, the abstract theorems may also be applied to many other non-linear boundary value problems to obtain sign-changing solutions, such as impulsive differential boundary value problems and multi-point differential boundary value problems.

2. Main results. Let $\left(X,\|\cdot\|_{X}\right)$ and $\left(Z,\|\cdot\|_{Z}\right)$ be the two real Banach spaces, and $P$ and $P_{1}$ normal cones of $X$ and $Z$, respectively. Denoted by $\leqslant$, the both partial orderings in $X$ and $Z$ are induced by the cones $P$ and $P_{1}$, respectively. For more discussions about cone and partial ordering we refer the reader to [20]. Let $u_{0}, v_{0} \in X$ and $\left[u_{0}, v_{0}\right]=\left\{x \in X \mid u_{0} \leqslant x \leqslant v_{0}\right\}$ denote an ordered interval of $X$. In this paper we will consider the existence of solutions of the operator equation

$$
L x=F x,
$$

where $L: \operatorname{dom} L \subset X \mapsto Z$ is a linear operator and $F: X \mapsto Z$ is a continuous and bounded operator.

Let $B: X \mapsto Z$ be a linear bounded operator such that $B: P \backslash\{\theta\} \rightarrow P_{1} \backslash\{\theta\}, e_{1} \in$ $P_{1} \backslash\{\theta\}, \bar{e}_{0}=L^{-1} e_{1} \in P \backslash\{\theta\}$. For any $x, y \in X$, denote by $x \prec_{0} y$ if there exists $\delta>0$ such that $y-x \geqslant \delta \bar{e}_{0}$. For any $x, y \in Z$, denote by $x \prec_{1} y$ if there exists $\delta>0$ such that $y-x \geqslant \delta e_{1}$. Let $\mathbb{N}$ denote the set of all natural numbers, and $\mathbb{N}^{+}=\{0\} \cup \mathbb{N}$.

First recall the concept of $\left(e_{1}, B\right)$-limit-increasing operator.

Definition 2.1. Let $D \subset X$ be a bounded and closed set, and $F: D \mapsto Z$ a bounded and continuous operator. Then $F$ is called an $\left(e_{1}, B\right)$-limit-increasing operator on the set $D$ if there exist a sequence of continuous operators $\left\{F_{n}\right\}_{n=1}^{\infty}$ and a sequence of positive numbers $\left\{M_{n}\right\}_{n=1}^{\infty}$ such that for all $n \in \mathbb{N}$,

$$
\begin{gathered}
-\frac{1}{n} e_{1} \leqslant F u-F_{n} u \leqslant \frac{1}{n} e_{1}, \quad \forall u \in D, \\
G_{n} v>G_{n} u, \quad \forall v, u \in D, v>u,
\end{gathered}
$$


where the operator $G_{n}: D \mapsto Z$ is defined by $G_{n} x=F_{n} x+M_{n} B x$ for all $x \in D$ and $n \in \mathbb{N}$. An $\left(e_{1}, B\right)$-limit-increasing operator $F$ on a bounded closed set $D$ whose interior is non-empty is called locally increasing at an interior point $x_{0}$ of $D$ if there exists $r_{0}>0$ such that $U\left(x_{0}, r_{0}\right) \subset D$ and

$$
F u=F_{1} u=F_{2} u=\cdots=F_{n} u=\cdots, \quad \forall u \in U\left(x_{0}, r_{0}\right) .
$$

REMARK 2.1. The concept of $\left(e_{1}, B\right)$-limit-increasing operator was put forward in $[13,18,19]$. However, there are some subtle differences between the concept of $\left(e_{1}, B\right)$-limit-increasing operator in this paper and those in $[13,18,19]$. In this paper, we will always assume that (2.2) and (2.3) hold if we assume that $F: D \mapsto Z$ is an $\left(e_{1}, B\right)$-limit-increasing operator.

Definition 2.2. ([15]) An operator $T: \mathscr{D}(T) \subset Z \mapsto X$ is called $e$-continuous at $x_{0} \in \mathscr{D}(T)$ if for every $\varepsilon>0$, there is number $\delta>0$, such that

$$
-\varepsilon e \leqslant T x-T x_{0} \leqslant \varepsilon e
$$

for every $x \in \mathscr{D}(T) \cap U\left(x_{0}, \delta\right)$. An operator $T$ is called $e$-continuous on $\mathscr{D}(T)$ if $T$ is $e$-continuous at every $x \in \mathscr{D}(T)$.

Definition 2.3. ([13]) Let $\alpha_{0}$ and $\beta_{0} \in X$. Then $\alpha_{0}$ and $\beta_{0}$ are said to be strict lower and upper solutions of (2.1), respectively, if $L \alpha_{0} \prec_{1} F \alpha_{0}$ and $F \beta_{0} \prec_{1} L \beta_{0}$.

DEFINITION 2.4. ([13]) Let $\alpha_{0}$ and $\beta_{0}$ be strict lower and upper solutions of (2.1), respectively. Then $\alpha_{0}$ and $\beta_{0}$ is called a pair of well-ordered strict lower and upper solutions of (2.1) if $\alpha_{0} \prec_{0} \beta_{0}$.

Definition 2.5. ([16]) Let $e \in P \backslash\{\theta\}$. An operator $T: \mathscr{D}(T) \subset Z \rightarrow X$ is said to be $e$-positive if for any $u \in P_{1} \backslash\{\theta\}$, there exist $\alpha=\alpha(u), \beta=\beta(u)>0$ such that

$$
\alpha e \leqslant T u \leqslant \beta e .
$$

DEFINITION 2.6. Let $\bar{x}$ be a non-zero solution of the operator equation (2.1). If $\bar{x} \in(-P)($ or $\bar{x} \in P$, or $\bar{x} \in E \backslash(P \cup(-P))$ ), then $\bar{x}$ is called a negative (or positive, or sign-changing) solution of (2.1).

From [14, Lemma 5.2] we have the following result.

LeMma 2.1. Let $E$ be an ordered Banach space with solid cone P. Let $K: E \mapsto E$ be a compact, e-positive, linear operator and let $F: E \mapsto E$ be a map such that for some $u_{0} \in E, u_{0}=K F\left(u_{0}\right)$. Suppose $F$ is Gâteaux differentiable at $u_{0}$ with strictly positive derivative $F^{\prime}\left(u_{0}\right)$. Denote by $r(T)$ the spectral radius of the operator $T=K F^{\prime}\left(u_{0}\right)$ and by $h_{0}$ the positive eigenfunction of $T$ corresponding to $r(T)$. Then there exists a $\tau_{0}>0$ such that for all $0<\tau<\tau_{0}$,

$$
r(T)>1 \text { implies }\left\{\begin{array}{l}
K F\left(u_{0}+\tau h_{0}\right)>u_{0}+\tau h_{0}, \\
K F\left(u_{0}-\tau h_{0}\right)<u_{0}-\tau h_{0},
\end{array}\right.
$$

and

$$
r(T)<1 \text { implies }\left\{\begin{array}{l}
K F\left(u_{0}+\tau h_{0}\right)<u_{0}+\tau h_{0}, \\
K F\left(u_{0}-\tau h_{0}\right)>u_{0}-\tau h_{0}
\end{array}\right.
$$

From [17, Theorem 19.2] we have the following Lemma 2.2. 
Lemma 2.2. (Krein-Rutman) Let $E$ be a Banach space, $P \subset E$ a total cone and $K \in L(E)$ a compact positive with $r(K)>0$. Then $r(K)$ is an eigenvalue with a positive eigenvector.

For convenience, let us introduce the following conditions to be used in the sequel.

$\left(H_{1}\right)$ There exists a pair of well-ordered strict lower and upper solutions $u_{0}$ and $v_{0}$ of (2.1) such that $u_{0}, v_{0} \in P$.

$\left(H_{2}\right) F$ is $\left(e_{1}, B\right)$-limit increasing on any bounded set of $X$ and is locally increasing at $\theta, F(\theta)=\theta, F$ is Fréchet differentiable at $\theta, F^{\prime}(\theta)=\beta_{0} B$, and $F$ is $e_{1}$-continuous on $\left[u_{0}, v_{0}\right]$, where $\beta_{0}>0$.

$\left(H_{3}\right)$ For each $M \geqslant 0, K_{M}:=(L+M B)^{-1}: Z \mapsto X$ exists and is completely continuous, $K_{M}$ is $\bar{e}_{M}$-continuous and $\bar{e}_{M}$-positive on any ordered interval of $Z$ for some $\bar{e}_{M} \in P \backslash\{\theta\}$; the algebraic multiplicity of each positive eigenvalue of $K_{M} B$ is 1 .

$\left(H_{4}\right)$ There exists a pair of well-ordered strict lower and upper solutions, $u_{1}$ and $v_{1}$, of (2.1) such that $u_{1}, v_{1} \in X \backslash(P \cup(-P))$ and $u_{0} \prec_{0} u_{1} \prec_{0} v_{1} \prec_{0} v_{0}$.

Assume in the sequel that the positive eigenvalues of $K B$ decreasingly are $\left\{\lambda_{n}\right\}_{n=1}^{\infty}$, where $K:=K_{0}$. Then we have the following main results.

THEOREM 2.1. Suppose that $\left(H_{1}\right)-\left(H_{3}\right)$ hold, $\beta_{0} \in\left(\frac{1}{\lambda_{2 n_{0}}}, \frac{1}{\lambda_{2 n_{0}+1}}\right)$, where $n_{0}$ is a natural number. Then (2.1) has at least one sign-changing solution. Moreover, (2.1) has at least one positive and one negative solution.

Proof. The proof is achieved in the following six steps:

Step 1. Since $F$ is an $\left(e_{1}, B\right)$-limit-increasing operator, which is locally increasing at $\theta$, there exist a sequence of continuous operators $\left\{F_{n}\right\}$, a sequence of positive numbers $\left\{M_{n}\right\}$ and $r_{0}>0$ such that (2.2)-(2.4) hold, where $x_{0}=\theta$ and $D=\left[u_{0}, v_{0}\right]$. For brevity, let us denote $F_{0}=F, G_{0}=F, M_{0}=0$ and $K_{n}=K_{M_{n}}$ for each $n \in \mathbb{N}$ in the sequel of this section. Now, since $u_{0}$ and $v_{0}$ are strict lower and upper solutions and $F$ is $e_{1}$-continuous on $\left[u_{0}, v_{0}\right]$, by (2.2) and the Definitions 2.1 and 2.3, for sufficiently large enough $n \in \mathbb{N}$ (assume without loss of generality that for all $n \in \mathbb{N}$ ), we have

$$
L u_{0} \prec_{1} F_{n} u_{0}, \quad F_{n} v_{0} \prec_{1} L v_{0} .
$$

Since $F^{\prime}(\theta)=\beta_{0} B$, we have

$$
\lim _{x \in X,\|x\|_{X} \rightarrow 0} \frac{\left\|F x-F \theta-\beta_{0} B x\right\|_{Z}}{\|x\|_{X}}=0 .
$$

By (2.4) we have for each $n \in \mathbb{N}^{+}$,

$$
\begin{gathered}
\lim _{x \in X,\|x\|_{X} \rightarrow 0} \frac{\left\|K_{n} G_{n} x-K_{n} G_{n} \theta-\left(\beta_{0}+M_{n}\right) K_{n} B x\right\|_{Z}}{\|x\|_{X}} \\
\leqslant \lim _{x \in X,\|x\|_{X} \rightarrow 0} \frac{\left\|K_{n}\right\|\left\|F x-F \theta-\beta_{0} B x\right\|_{Z}}{\|x\|_{X}}=0 .
\end{gathered}
$$

This implies that $K_{n} G_{n}$ is Fréchet differentiable at $\theta$ for each $n \in \mathbb{N}^{+}$, and $\left(K_{n} G_{n}\right)^{\prime}(\theta)=\left(\beta_{0}+M_{n}\right) K_{n} B$. Let $\mu$ be a positive eigenvalue of the linear operator $\left(\beta_{0}+M_{n}\right) K_{n} B$ and $x_{\mu}$ be the corresponding eigenfunction, that is

$$
\left(\beta_{0}+M_{n}\right) K_{n} B x_{\mu}=\mu x_{\mu} .
$$


Then, we have

$$
\left(\beta_{0}+M_{n}\right) B x_{\mu}=\mu\left(L+M_{n} B\right) x_{\mu},
$$

and so

$$
K B x_{\mu}=\frac{\mu}{\beta_{0}+(1-\mu) M_{n}} x_{\mu} .
$$

Since the positive eigenvalues of $K B$ are $\left\{\lambda_{i}\right\}$, there exists $i_{0}$ such that

$$
\lambda_{i_{0}}=\frac{\mu}{\beta_{0}+(1-\mu) M_{n}},
$$

and so

$$
\mu=\frac{\lambda_{i_{0}}\left(\beta_{0}+M_{n}\right)}{1+\lambda_{i_{0}} M_{n}} .
$$

On other hand, we can easily prove that every positive number $\mu$ satisfying (2.5) is an eigenvalue of the linear operator $\left(\beta_{0}+M_{n}\right) K_{n} B$. Thus, the sequence of positive eigenvalues of the linear operator $\left(\beta_{0}+M_{n}\right) K_{n} B$ is

$$
\left\{\frac{\lambda_{i}\left(\beta_{0}+M_{n}\right)}{1+\lambda_{i} M_{n}}\right\}_{i=1}^{\infty} .
$$

Step 2. For each $n \in \mathbb{N}^{+}$, let

$$
\begin{aligned}
& S_{n}^{+}=\left\{x \in\left[u_{0}, v_{0}\right] \mid x>\theta, x=K_{n} G_{n} x\right\}, \\
& S_{n}^{-}=\left\{x \in\left[u_{0}, v_{0}\right] \mid x<\theta, x=K_{n} G_{n} x\right\} .
\end{aligned}
$$

By $\left(\mathrm{H}_{3}\right)$, there exists $\bar{e}_{n} \in P \backslash\{\theta\}$ such that, for each $n \in \mathbb{N}^{+}, K_{n} B$ is $\bar{e}_{n}$-positive and $\bar{e}_{n}$-continuous. Now we shall show that for each $n \in \mathbb{N}^{+}$, there exists $\zeta_{n}>0$ such that

$$
S_{n}^{+} \geqslant \zeta_{n} \bar{e}_{n}, \quad S_{n}^{-} \leqslant-\zeta_{n} \bar{e}_{n}
$$

Let $x_{0} \in S_{n}^{+}$. Now, since $G_{n} x_{0}>G_{n} \theta=\theta$ and $K_{n}$ is $\bar{e}_{n}$-positive, there exists $\beta_{x_{0}}^{(n)}>0$ such that $x_{0}=K_{n} G_{n} x_{0} \geqslant \beta_{x_{0}}^{(n)} \bar{e}_{n}$. On the other hand, since $G_{n}: X \mapsto Z$ is continuous and $K_{n}: Z \mapsto X$ is $\bar{e}_{n}$-continuous, for $\frac{\beta_{x_{0}}^{(n)}}{2}>0$, there exits $r_{x_{0}}>0$ such that for any $x \in U\left(x_{0}, r_{x_{0}}\right) \cap S_{n}^{+}$,

$$
-\frac{1}{2} \beta_{x_{0}}^{(n)} \bar{e}_{n} \leqslant K_{n} G_{n} x-K_{n} G_{n} x_{0} \leqslant \frac{1}{2} \beta_{x_{0}}^{(n)} \bar{e}_{n},
$$

and thus, for any $x \in U\left(x_{0}, r_{x_{0}}\right) \cap S_{n}^{+}$, we have

$$
x \geqslant x_{0}-\frac{1}{2} \beta_{x_{0}}^{(n)} \bar{e}_{n} \geqslant \frac{1}{2} \beta_{x_{0}}^{(n)} \bar{e}_{n} .
$$

Obviously, $\left\{U\left(x_{0}, r_{x_{0}}\right) \mid x_{0} \in S_{n}^{+}\right\}$is an open cover of $S_{n}^{+}$. Since $P \subset X$ and $P_{1} \subset Z$ are normal, (2.3) implies that $G_{n}$ is bounded on $\left[u_{0}, v_{0}\right]$ for each $n \in \mathbb{N}$. Thus, $S_{n}^{+}=K_{n} G_{n} S_{n}^{+} \subset X$ is a relative compact set for each $n \in \mathbb{N}^{+}$(note $G_{0}=F$ also 
is bounded). Therefore, there exist finite subsets of $\left\{U\left(x_{0}, r_{x_{0}}\right) \mid x_{0} \in S_{n}^{+}\right\}$, say, $U\left(x_{1}, r_{x_{1}}\right), \ldots, U\left(x_{k_{n}}, r_{k_{n}}\right)$, such that

$$
S_{n}^{+} \subset \bigcup_{i=1}^{k_{n}} U\left(x_{i}, r_{x_{i}}\right) .
$$

Let $\beta_{+}^{(n)}=\min \left\{\frac{1}{2} \beta_{x_{1}}^{(n)}, \frac{1}{2} \beta_{x_{2}}^{(n)}, \ldots, \frac{1}{2} \beta_{x_{k_{n}}}^{(n)}\right\}>0$ for each $n \in \mathbb{N}^{+}$. Then, we have $S_{n}^{+} \geqslant \beta_{+}^{(n)} \bar{e}_{n}$. Similarly, there exists $\beta_{-}^{(n)}>0$ such that $S_{n}^{-} \leqslant-\beta_{-}^{(n)} \bar{e}_{n}$. Let $\beta^{(n)}=$ $\frac{1}{2} \min \left\{\beta_{+}^{(n)}, \beta_{-}^{(n)}\right\}$, then (2.6) holds.

Step 3. Let $n \in \mathbb{N}^{+}$be fixed at present. The spectrum radius of $\left(K_{n} G_{n}\right)^{\prime}(\theta)=\left(\beta_{0}+\right.$ $\left.M_{n}\right) K_{n} B$ is

$$
r\left(\left(\beta_{0}+M_{n}\right) K_{n} B\right)=\frac{\lambda_{1}\left(\beta_{0}+M_{n}\right)}{1+\lambda_{1} M_{n}}>1
$$

By Lemma 2.2, there exists $h_{n} \in P \backslash\{\theta\}$ such that

$$
\left(\beta_{0}+M_{n}\right) K_{n} B h_{n}=r\left(\left(\beta_{0}+M_{n}\right) K_{n} B\right) h_{n} .
$$

Since $K_{n}$ is $\bar{e}_{n}$-positive and $B: P \backslash\{\theta\} \mapsto P_{1} \backslash\{\theta\}$, by (2.7), there exist $\alpha\left(h_{n}\right)>0$ and $\beta\left(h_{n}\right)>0$ such that

$$
\alpha\left(h_{n}\right) \bar{e}_{n} \leqslant h_{n} \leqslant \beta\left(h_{n}\right) \bar{e}_{n} .
$$

By Lemma 2.1, there exists $\tau_{n}>0$ such that for any $\tau \in\left(0, \tau_{n}\right]$,

$$
-\tau h_{n}>K_{n} G_{n}\left(-\tau h_{n}\right), \quad K_{n} G_{n}\left(\tau h_{n}\right)>\tau h_{n} .
$$

By (2.6), (2.8) and (2.9), we may take $\tau_{n}>0$ small enough such that

$$
\begin{gathered}
u_{0}<v_{2, n}<\theta<u_{2, n}<v_{0}, \\
S_{n}^{+} \geqslant u_{2, n}, S_{n}^{-} \leqslant v_{2, n}
\end{gathered}
$$

and

$$
u_{2, n}<K_{n} G_{n} u_{2, n}, K_{n} G_{n} v_{2, n}<v_{2, n},
$$

where $u_{2, n}=\tau_{n} h_{n}$ and $v_{2, n}=-\tau_{n} h_{n}$. Since 1 is not an eigenvalue of the linear operator $\left(K_{n} G_{n}\right)^{\prime}(\theta)$, there exists $\bar{r}_{n}>0$ such that $K_{n} G_{n}$ has the unique fixed point $\theta$ in $U\left(\theta, \bar{r}_{n}\right)$, and

$$
\operatorname{deg}\left(I-K_{n} G_{n}, U\left(\theta, \bar{r}_{n}\right), \theta\right)=(-1)^{2 n_{0}}=1 .
$$

Assume that $\bar{r}_{n}>0$ small enough such that $v_{2, n}, u_{2, n} \notin U\left(\theta, \bar{r}_{n}\right), U\left(\theta, \bar{r}_{n}\right) \subset\left[u_{0}, v_{0}\right]$ and $\bar{r}_{n}<\bar{r}_{0}<r_{0}$.

Step 4. For each $n \in \mathbb{N}$, let

$$
\begin{aligned}
& \Omega_{1, n}=\left\{x \in\left[u_{0}, v_{0}\right] \mid \text { there exists } \tau>0 \text { such that } K_{n} G_{n} x \leqslant K_{n} G_{n} v_{2, n}-\tau \bar{e}_{n}\right\}, \\
& \Omega_{2, n}=\left\{x \in\left[u_{0}, v_{0}\right] \mid \text { there exists } \tau>0 \text { such that } K_{n} G_{n} x \geqslant K_{n} G_{n} u_{2, n}+\tau \bar{e}_{n}\right\} .
\end{aligned}
$$


It is easy to see that $u_{0} \in \Omega_{1, n}$ and $v_{0} \in \Omega_{2, n}$. By the $\bar{e}_{n}$-continuity of $K_{n} G_{n}$, it is easy to see that $\Omega_{1, n}$ and $\Omega_{2, n}$ are open subsets of $\left[u_{0}, v_{0}\right]$. Now assume that $n$ is a fixed natural number. Now we shall show that for any $x \in \partial_{\left[u_{0}, v_{0}\right]} \Omega_{1, n}$ and $t \in[0,1]$,

$$
x \neq t K_{n} G_{n} x+(1-t) u_{0} .
$$

If (2.14) is not true, then there exists $x_{0} \in \partial_{\left[u_{0}, v_{0}\right]} \Omega_{1, n}$ and $t_{0} \in[0,1]$ such that

$$
x_{0}=t_{0} K_{n} G_{n} x_{0}+\left(1-t_{0}\right) u_{0} .
$$

Notice that $x_{0} \in \partial_{\left[u_{0}, v_{0}\right]} \Omega_{1, n}$, so we have

$$
K_{n} G_{n} x_{0} \leqslant K_{n} G_{n} v_{2, n}<v_{2, n} .
$$

It follows from (2.10) and (2.15) that $x_{0}<v_{2, n}$. Thus, by the $\bar{e}_{n}$-positivity of $K_{n}$ we have

$$
K_{n} G_{n} v_{2, n}-K_{n} G_{n} x_{0}=K_{n}\left(G_{n} v_{2, n}-G_{n} x_{0}\right) \geqslant \alpha\left(G_{n} v_{2, n}-G_{n} x_{0}\right) \bar{e}_{n},
$$

where $\alpha\left(G_{n} v_{2, n}-G_{n} x_{0}\right)>0$. This implies that $x_{0} \in \Omega_{1, n}$, which contradicts $x_{0} \in$ $\partial_{\left[u_{0}, v_{0}\right]} \Omega_{1, n}$. Thus, (2.14) holds, and so

$$
i\left(K_{n} G_{n}, \Omega_{1, n},\left[u_{0}, v_{0}\right]\right)=1 .
$$

Similarly, we have

$$
i\left(K_{n} G_{n}, \Omega_{2, n},\left[u_{0}, v_{0}\right]\right)=1 .
$$

Obviously, we have

$$
i\left(K_{n} G_{n},\left[u_{0}, v_{0}\right],\left[u_{0}, v_{0}\right]\right)=1 .
$$

From (2.3.5) of [20], we have

$$
i\left(K_{n} G_{n}, U\left(\theta, \bar{r}_{n}\right),\left[u_{0}, v_{0}\right]\right)=\operatorname{deg}\left(I-K_{n} G_{n} \cdot r, U\left(\theta, \bar{R}_{n}\right) \cap r^{-1}\left(U\left(\theta, \bar{r}_{n}\right)\right), \theta\right),
$$

where $r: X \mapsto\left[u_{0}, v_{0}\right]$ is a retraction and $\bar{R}_{n}>\bar{r}_{0}$. It is easy to see that each fixed point of $K_{n} G_{n} \cdot r$ in $U\left(\theta, \bar{R}_{n}\right) \cap r^{-1}\left(U\left(\theta, \bar{r}_{n}\right)\right)$ must belong to $U\left(\theta, \bar{r}_{n}\right)$. Thus, by the properties of the Leray-Schauder degree,

$$
\operatorname{deg}\left(I-K_{n} G_{n}, U\left(\theta, \bar{r}_{n}\right), \theta\right)=\operatorname{deg}\left(I-K_{n} G_{n} \cdot r, U\left(\theta, \bar{R}_{n}\right) \cap r^{-1}\left(U\left(\theta, \bar{r}_{n}\right)\right), \theta\right) .
$$

It follows from (2.13), (2.19) and (2.20) that

$$
i\left(K_{n} G_{n}, U\left(\theta, \bar{r}_{n}\right),\left[u_{0}, v_{0}\right]\right)=1 .
$$

By (2.16)-(2.18) and (2.21), we have

$$
i\left(K_{n} G_{n},\left[u_{0}, v_{0}\right] \backslash\left(C l_{\left[u_{0}, v_{0}\right]} \Omega_{1, n} \cup C l_{\left[u_{0}, v_{0}\right]} \Omega_{2, n} \cup \bar{U}\left(\theta, \bar{r}_{n}\right)\right),\left[u_{0}, v_{0}\right]\right)=-2 .
$$

By (2.16), (2.17) and (2.22), $K_{n} G_{n}$ has three fixed points $x_{1, n} \in \Omega_{1, n}, x_{2, n} \in \Omega_{2, n}$ and $x_{3, n} \in\left[u_{0}, v_{0}\right] \backslash\left(C l_{\left[u_{0}, v_{0}\right]} \Omega_{1, n} \cup C l_{\left[u_{0}, v_{0}\right]} \Omega_{2, n} \cup \bar{U}\left(\theta, \bar{r}_{n}\right)\right)$, respectively. Let us show that

$$
\left\|x_{i, n}\right\|_{X} \geqslant \bar{r}_{0}, \quad i=1,2,3 .
$$


We only show that $\left\|x_{3, n}\right\|_{X} \geqslant \bar{r}_{0}$. By contradiction, assume that $\left\|x_{3, n}\right\|_{X}<\bar{r}_{0}<r_{0}$. Since

$$
x_{3, n}=K_{n} G_{n} x_{3, n}=\left(L+M_{n}\right)^{-1}\left(F_{n} x_{3, n}+M_{n} B x_{3, n}\right),
$$

we have $x_{3, n}=K F x_{3, n}$. Now, since $\theta$ is the unique solution of $K F$ in $U\left(\theta, \bar{r}_{0}\right)$, we see that $x_{3, n}=\theta$, which contradicts $x_{3, n} \notin \bar{B}\left(\theta, \bar{r}_{n}\right)$, and so (2.23) holds.

Step 5. Since $\left[u_{0}, v_{0}\right]$ is bounded and $F:\left[u_{0}, v_{0}\right] \mapsto Z$ is bounded, there exists $R^{\prime}>0$ such that $\left\|F x_{1, n}\right\|_{Z}<R^{\prime}$. It follows from (2.2) that

$$
\theta \leqslant F_{n} x_{1, n}-F x_{1, n}+\frac{1}{n} e_{1} \leqslant \frac{2}{n} e_{1} \leqslant 2 e_{1},
$$

and so

$$
\begin{aligned}
\left\|F_{n} x_{1, n}\right\|_{Z} & \leqslant\left\|F_{n} x_{1, n}-F x_{1, n}+\frac{1}{n} e_{1}\right\|_{Z}+\left\|F x_{1, n}-\frac{1}{n} e_{1}\right\|_{Z} \\
& \leqslant 2 \gamma_{0}\left\|e_{1}\right\|_{Z}+\left\|F x_{1, n}-\frac{1}{n} e_{1}\right\|_{Z} \\
& \leqslant 2 \gamma_{0}\left\|e_{1}\right\|_{Z}+\left\|F x_{1, n}\right\|_{Z}+\left\|e_{1}\right\|_{Z} \\
& \leqslant\left(2 \gamma_{0}+1\right)\left\|e_{1}\right\|_{Z}+R^{\prime}
\end{aligned}
$$

where $\gamma_{0}>0$ is the normal constant of the cone $P_{1}$. Since $x_{1, n}=K F_{n} x_{1, n}$ and $K$ is a linear completely continuous operator, $\left\{x_{1, n}\right\}_{n=1}^{\infty}$ is a relatively compact set. Thus, there exist a sub-sequence of $\left\{x_{1, n}\right\}_{n=1}^{\infty}$ (assume without loss of generality that the sub-sequence is $\left\{x_{1, n}\right\}_{n=1}^{\infty}$ itself $)$ and $x_{1}^{*}$ such that $x_{1, n} \rightarrow x_{1}^{*}(n \rightarrow \infty)$. Since

$$
-\frac{1}{n} e_{1} \leqslant F_{n} x_{1, n}-F x_{1, n} \leqslant \frac{1}{n} e_{1}
$$

we have $F_{n} x_{1, n}-F x_{1, n} \rightarrow \theta(n \rightarrow \infty)$ and so $K\left(F_{n} x_{1, n}-F x_{1, n}\right) \rightarrow \theta(n \rightarrow \infty)$. Consequently, we have

$$
\begin{aligned}
K F x_{1, n} & =K\left(F x_{1, n}-F_{n} x_{1, n}\right)+K F_{n} x_{1, n} \\
& =K\left(F x_{1, n}-F_{n} x_{1, n}\right)+x_{1, n} \rightarrow x_{1}^{*}(n \rightarrow \infty) .
\end{aligned}
$$

On the other hand, $K F x_{1, n} \rightarrow K F x_{1}^{*}(n \rightarrow \infty)$. Therefore, $x_{1}^{*}=K F x_{1}^{*}$, and so $L x_{1}^{*}=$ $F x_{1}^{*}$, that is, $x_{1}^{*}$ is a solution of (2.1). Similarly, there are two sub-sequences of $\left\{x_{2, n}\right\}_{n=1}^{\infty}$ and $\left\{x_{3, n}\right\}_{n=1}^{\infty}$ (assume that the two sub-sequences are themselves) and $x_{2}^{*}$, $x_{3}^{*}$, such that $x_{2, n} \rightarrow x_{2}^{*}$ and $x_{3, n} \rightarrow x_{3}^{*}(n \rightarrow \infty)$. Then, $x_{2}^{*}$ and $x_{3}^{*}$ are two solutions of (2.1).

Step 6. Note that $x_{1, n} \in \Omega_{1, n}$, so we have

$$
x_{1, n}=K_{n} G_{n} x_{1, n} \leqslant K_{n} G_{n} v_{2, n}-\tau \bar{e}_{n}<v_{2, n}<\theta
$$

for some $\tau>0$, and so $x_{1}^{*} \leqslant \theta$. It follows from (2.23) that $\left\|x_{1}^{*}\right\|_{X} \geqslant \bar{r}_{0}$. Thus, $x_{1}^{*}$ is a negative solution of (2.1). Similarly, $x_{2}^{*}$ is a positive solution of (2.1). Finally we shall show that $x_{3}^{*}$ is a sign-changing solution of (2.1). By contradiction, assume that $x_{3}^{*}$ is not a sign-changing solution of (2.1). It follows from (2.23) that $x_{3}^{*}$ is not a zero solution of (2.1). Assume that $x_{3}^{*}$ is a positive solution of (2.1), then by (2.6) we have

$$
x_{3}^{*} \geqslant \zeta_{0} \bar{e}_{0}
$$


It is easy to see that

$$
\begin{aligned}
x_{3, n}-x_{3}^{*} & =K F_{n} x_{3, n}-K F x_{3}^{*} \\
& =K\left(F_{n} x_{3, n}-F x_{3, n}\right)+K\left(F x_{3, n}-F x_{3}^{*}\right) \\
& \geqslant-\frac{1}{n} K e_{1}+K\left(F x_{3, n}-F x_{3}^{*}\right) \\
& =-\frac{1}{n} \bar{e}_{0}+K\left(F x_{3, n}-F x_{3}^{*}\right) .
\end{aligned}
$$

Since $\frac{1}{n} \rightarrow 0, x_{3, n} \rightarrow x_{3}^{*}(n \rightarrow \infty)$ and $K$ is $\bar{e}_{0}$-continuous, for $\frac{\zeta_{0}}{2}>0$, there exists $n_{1}>0$ large enough such that

$$
-\frac{1}{n} \bar{e}_{0}+K\left(F x_{3, n_{1}}-F x_{3}\right) \geqslant-\frac{1}{2} \zeta_{0} \bar{e}_{0} .
$$

It follows from (2.24)-(2.26) that

$$
x_{3, n_{1}} \geqslant x_{3}^{*}-\frac{1}{2} \zeta_{0} \bar{e}_{0} \geqslant \frac{1}{2} \zeta_{0} \bar{e}_{0},
$$

that is, $x_{3, n_{1}} \in S_{n_{1}}^{+}$. From (2.11), we have for any $x \in S_{n}^{+}$and $n \in \mathbb{N}$,

$$
x=K_{n} G_{n} x \geqslant K_{n} G_{n} u_{2, n}>u_{2, n},
$$

and so by (2.2) and the $\bar{e}_{n}$-positive property of $K_{n}$, we have

$$
K_{n} G_{n} x-K_{n} G_{n} u_{2, n} \geqslant \alpha\left(G_{n} x-G_{n} u_{2, n}\right) \bar{e}_{n},
$$

where $\alpha\left(G_{n} x-G_{n} u_{2, n}\right)>0$. This implies that $x \in \Omega_{2, n}$, and so $S_{n}^{+} \subset \Omega_{2, n}$ for each $n \in \mathbb{N}$. Similarly, we have $S_{n}^{-} \subset \Omega_{1, n}$ for each $n \in \mathbb{N}$. Hence,

$$
x_{3, n_{1}} \in\left[u_{0}, v_{0}\right] \backslash\left(C l_{\left[u_{0}, v_{0}\right]} \Omega_{1, n_{1}} \cup C l_{\left[u_{0}, v_{0}\right]} \Omega_{2, n_{1}} \cup \bar{U}\left(\theta, \bar{r}_{n_{1}}\right)\right) \subset\left[u_{0}, v_{0}\right] \backslash\left(S_{n_{1}}^{+} \cup S_{n_{1}}^{-}\right),
$$

which is a contradiction. Therefore, $x_{3}^{*}$ is a sign-changing solution of (2.1). The proof is complete.

THEOREM 2.2. Suppose that $\left(H_{1}\right)-\left(H_{4}\right)$ hold, $\beta_{0}>\frac{1}{\lambda_{1}}, \beta_{0} \neq \frac{1}{\lambda_{n}}$ for all $n \geqslant 2$. Then (2.1) has at least four sign-changing solutions. Moreover, (2.1) has at least one positive and one negative solution.

Proof. In a similar way as that of Theorem 2.1, take a sequence of continuous operators $\left\{F_{n}\right\}$, a sequence of positive numbers $\left\{M_{n}\right\}$ and $r_{0}>0$ such that (2.2)-(2.4) hold. Let $S_{n}^{+}$and $S_{n}^{-}$be defined as in Theorem 2.1. Then there exists $\zeta_{n}>0$ such that (2.6) holds. A similar argument as in Theorem 2.1 shows that $K_{n} G_{n}$ is Fréchet differentiable at $\theta$, and $r\left(\left(K_{n} G_{n}\right)^{\prime}(\theta)\right)>1$. Thus, there exist $u_{2, n}, v_{2, n}$ such that (2.10)(2.12) hold. For each $n \in \mathbb{N}^{+}$, take $0<\bar{r}_{n}<\bar{r}_{0}<r_{0}$ small enough such that $u_{2, n}, v_{2, n} \notin$ $U\left(\theta, \bar{r}_{n}\right), U\left(\theta, \bar{r}_{n}\right) \subset\left[u_{0}, v_{0}\right], K_{n} G_{n}$ has the unique fixed point $\theta$ in $U\left(\theta, \bar{r}_{n}\right)$, and

$$
i\left(K_{n} G_{n}, U\left(\theta, \bar{r}_{n}\right),\left[u_{0}, v_{0}\right]\right)=\operatorname{deg}\left(I-K_{n} G_{n}, U\left(\theta, \bar{r}_{n}\right), \theta\right)=(-1)^{k}= \pm 1,
$$


where $k$ is the sum of all algebraic multiplicities of eigenvalues of $\left(K_{n} G_{n}\right)^{\prime}(\theta)$ larger than 1 . Since $F$ is $e_{1}$-continuous, we may take $\delta_{0}>0$ small enough such that $\widetilde{u}_{1} \prec_{0} \widetilde{v}_{1}$, and

$$
L \widetilde{u}_{1} \prec_{1} F \widetilde{u}_{1}, \quad F \widetilde{v}_{1} \prec_{1} L \widetilde{v}_{1} .
$$

where $\widetilde{u}_{1}=u_{1}+\delta_{0} \bar{e}_{0}$ and $\widetilde{v}_{1}=v_{1}-\delta_{0} \bar{e}_{0}$. This means that $\widetilde{u}_{1}$ and $\widetilde{v}_{1}$ is a pair of wellordered strict lower and upper solutions of (2.1). Assume without loss of generality that for all $n \in \mathbb{N}$,

$$
\begin{aligned}
& L \widetilde{u}_{1} \prec_{1} F_{n} \widetilde{u}_{1}, \quad F_{n} \widetilde{v}_{1} \prec_{1} L \widetilde{v}_{1}, \\
& L u_{1} \prec_{1} F_{n} u_{1}, \quad F_{n} v_{1} \prec_{1} L v_{1}, \\
& L u_{0} \prec_{1} F_{n} u_{0}, \quad F_{n} v_{0} \prec_{1} L v_{0} .
\end{aligned}
$$

For each $n \in \mathbb{N}$, let

$$
\begin{aligned}
& O_{1, n}=\left\{x \in\left[u_{0}, v_{0}\right] \mid \text { there exists } \tau>0 \text { such that } K_{n} G_{n} x \geqslant K_{n} G_{n} \widetilde{u}_{1}+\tau \bar{e}_{n}\right\}, \\
& \Omega_{2, n}=\left\{x \in\left[u_{0}, v_{0}\right] \mid \text { there exists } \tau>0 \text { such that } K_{n} G_{n} x \geqslant K_{n} G_{n} u_{2, n}+\tau \bar{e}_{n}\right\}, \\
& \Omega_{3, n}=\left\{x \in\left[u_{0}, v_{0}\right] \mid \text { there exists } \tau>0 \text { such that } K_{n} G_{n} x \leqslant K_{n} G_{n} v_{2, n}-\tau \bar{e}_{n}\right\}, \\
& O_{4, n}=\left\{x \in\left[u_{0}, v_{0}\right] \mid \text { there exists } \tau>0 \text { such that } K_{n} G_{n} x \leqslant K_{n} G_{n} \widetilde{v}_{1}-\tau \bar{e}_{n}\right\}, \\
& \Omega_{1, n}=\left\{x \in\left[u_{0}, v_{0}\right] \mid \text { there exists } \tau>0 \text { such that } K_{n} G_{n} x \geqslant K_{n} G_{n} u_{1}+\tau \bar{e}_{n}\right\}, \\
& \Omega_{4, n}=\left\{x \in\left[u_{0}, v_{0}\right] \mid \text { there exists } \tau>0 \text { such that } K_{n} G_{n} x \leqslant K_{n} G_{n} v_{1}-\tau \bar{e}_{n}\right\} .
\end{aligned}
$$

Similar to the proof of Theorem 2.1, we have

$$
\begin{gathered}
i\left(K_{n} G_{n}, \Omega_{i, n},\left[u_{0}, v_{0}\right]\right)=1, \quad i=1,2,3,4, n \in \mathbb{N}, \\
i\left(K_{n} G_{n}, O_{i, n},\left[u_{0}, v_{0}\right]\right)=1, \quad i=1,4, n \in \mathbb{N} .
\end{gathered}
$$

Obviously, $v_{0} \in O_{1, n} \cap \Omega_{2, n}$. Next we shall show that for any $x \in \partial_{\left[u_{0}, v_{0}\right]}\left(O_{1, n} \cap \Omega_{2, n}\right)$ and $t \in[0,1]$,

$$
x \neq t K_{n} G_{n} x+(1-t) v_{0},
$$

Suppose this is not the case. Then there exists $x_{0} \in \partial_{\left[u_{0}, v_{0}\right]}\left(O_{1, n} \cap \Omega_{2, n}\right)$ and $t_{0} \in[0,1]$ such that $x_{0}=t_{0} K_{n} G_{n} x+\left(1-t_{0}\right) v_{0}$. Now we have three cases: (i) $x_{0} \in\left(\partial_{\left[u_{0}, v_{0}\right]} O_{1, n}\right) \cap$ $\Omega_{2, n}$; (ii) $x_{0} \in O_{1, n} \cap \partial_{\left[u_{0}, v_{0}\right]} \Omega_{2, n}$; (iii) $x_{0} \in \partial_{\left[u_{0}, v_{0}\right]} O_{1, n} \cap \partial_{\left[u_{0}, v_{0}\right]} \Omega_{2, n}$. Similar to the proof of (2.14) in Theorem 2.1, we can get contradictions for the above three cases, and so (2.33) holds. Thus, we have

$$
i\left(K_{n} G_{n}, O_{1, n} \cap \Omega_{2, n},\left[u_{0}, v_{0}\right]\right)=i\left(v_{0}, O_{1, n} \cap \Omega_{2, n},\left[u_{0}, v_{0}\right]\right)=1 .
$$

Similarly, we have

$$
i\left(K_{n} G_{n}, O_{1, n} \cap O_{4, n},\left[u_{0}, v_{0}\right]\right)=i\left(\frac{\widetilde{u}_{1}+\widetilde{v}_{1}}{2}, O_{1, n} \cap O_{4, n},\left[u_{0}, v_{0}\right]\right)=1,
$$




$$
\begin{array}{r}
i\left(K_{n} G_{n}, O_{4, n} \cap \Omega_{3, n},\left[u_{0}, v_{0}\right]\right)=i\left(u_{0}, O_{4, n} \cap \Omega_{3, n},\left[u_{0}, v_{0}\right]\right)=1, \\
i\left(K_{n} G_{n}, O_{1, n} \cap \Omega_{4, n},\left[u_{0}, v_{0}\right]\right)=i\left(\frac{\widetilde{u}_{1}+v_{1}}{2}, O_{1, n} \cap \Omega_{4, n},\left[u_{0}, v_{0}\right]\right)=1, \\
i\left(K_{n} G_{n}, O_{4, n} \cap \Omega_{1, n},\left[u_{0}, v_{0}\right]\right)=i\left(\frac{u_{1}+\widetilde{v}_{1}}{2}, O_{4, n} \cap \Omega_{1, n},\left[u_{0}, v_{0}\right]\right)=1 .
\end{array}
$$

Let

$$
\begin{aligned}
& D_{1, n}=O_{1, n} \backslash\left(C l_{\left[u_{0}, v_{0}\right]}\left(O_{1, n} \cap \Omega_{4, n}\right) \cup C l_{\left[u_{0}, v_{0}\right]}\left(O_{1, n} \cap \Omega_{2, n}\right)\right), \\
& D_{2, n}=O_{4, n} \backslash\left(C l_{\left[u_{0}, v_{0}\right]}\left(O_{4, n} \cap \Omega_{1, n}\right) \cup C l_{\left[u_{0}, v_{0}\right]}\left(O_{4, n} \cap \Omega_{3, n}\right)\right) .
\end{aligned}
$$

It follows from (2.32)-(2.34) and (2.36)-(2.38) that

$$
\begin{aligned}
& i\left(K_{n} G_{n}, D_{1, n},\left[u_{0}, v_{0}\right]\right)=-1, \\
& i\left(K_{n} G_{n}, D_{2, n},\left[u_{0}, v_{0}\right]\right)=-1 .
\end{aligned}
$$

By (2.34)-(2.40), $K_{n} G_{n}$ has fixed points $x_{1, n} \in O_{1, n} \cap \Omega_{2, n}, x_{2, n} \in O_{4, n} \cap \Omega_{3, n}, x_{3, n} \in$ $O_{1, n} \cap O_{4, n}, x_{4, n} \in D_{1, n}, x_{5, n} \in D_{2, n}$. Similar to the proof of (2.23) in Theorem 2.1, we have

$$
\left\|x_{i, n}\right\|_{X} \geqslant \bar{r}_{0}, i=1,2,3,4,5, n \in \mathbb{N} \text {. }
$$

A similar argument as in Step 5 of Theorem 2.1 shows that for each $i=1,2,3,4,5$ there are sub-sequences of $\left\{x_{i, n}\right\}_{n=1}^{\infty}$ (assume without loss of generality that the four sub-sequences are $\left\{x_{i, n}\right\}_{n=1}^{\infty}$ themselves) and $x_{i}^{*}$ such that $x_{i, n} \rightarrow x_{i}^{*}(n \rightarrow \infty)$. Then, $x_{i}^{*}$ is a solution of (2.1) for each $i=1,2,3,4,5$. Moreover, by a similar argument as in Step 6 of Theorem 2.1, we see that $x_{1}^{*}$ is a positive solution of $(2.1), x_{2}^{*}$ is a negative solution of (2.1) and $x_{3}^{*}, x_{4}^{*}$ and $x_{5}^{*}$ are three sign-changing solutions of (2.1). Since $x_{3, n} \in O_{1, n} \cap O_{4, n}$, there exists $\tau>0$ such that

$$
x_{3, n}=K_{n} G_{n} x_{3, n} \leqslant K_{n} G_{n} \widetilde{v}_{1}-\tau \bar{e}_{n}<K_{n} G_{n} \widetilde{v}_{1}<\widetilde{v}_{1}
$$

and so $x_{3}^{*} \leqslant \widetilde{v}_{1}$. Next we shall show that $x_{3}^{*} \neq x_{4}^{*}$. Suppose this is not the case. Then $x_{4}^{*} \leqslant \widetilde{v}_{1}=v_{1}-\delta_{0} \bar{e}_{0}$. On the other hand, a similar argument as in Step 6 of Theorem 2.1 shows that

$$
\begin{aligned}
x_{4, n}-x_{4}^{*} & =K F_{n} x_{4, n}-K F x_{4}^{*} \\
& =K\left(F_{n} x_{4, n}-F x_{4, n}\right)+K\left(F x_{4, n}-F x_{4}^{*}\right) \\
& \leqslant \frac{1}{n} K e_{1}+K\left(F x_{4, n}-F x_{4}^{*}\right) \\
& =\frac{1}{n} \bar{e}_{0}+K\left(F x_{4, n}-F x_{4}^{*}\right) .
\end{aligned}
$$

Since $F$ is $e_{1}$-continuous and $\frac{1}{n} \rightarrow 0(n \rightarrow+\infty)$, there exists $n_{1}>0$ large enough such that

$$
\frac{1}{n} \bar{e}_{0}+K\left(F x_{4, n_{1}}-F x_{4}^{*}\right) \leqslant \frac{1}{2} \delta_{0} \bar{e}_{0}
$$


Then, by (2.41) we have

$$
x_{4, n_{1}} \leqslant x_{4}^{*}+\frac{1}{2} \delta_{0} \bar{e}_{0} \leqslant v_{1}-\frac{1}{2} \delta_{0} \bar{e}_{0}<v_{1} .
$$

Since $K_{n_{1}}$ is $\bar{e}_{n_{1}}$-positive and $G_{n_{1}}$ is strictly increasing, we have

$$
K_{n_{1}} G_{n_{1}} x_{4, n_{1}} \leqslant K_{n_{1}} G_{n_{1}} v_{1}-\alpha\left(G_{n_{1}} v_{1}-G_{n_{1}} x_{4, n_{1}}\right) \bar{e}_{n_{1}}
$$

where $\alpha\left(G_{n_{1}} v_{1}-G_{n_{1}} x_{4, n_{1}}\right)>0$. This implies that $x_{4, n_{1}} \in \Omega_{4, n_{1}}$, which contradicts $x_{4, n_{1}} \in$ $D_{1, n_{1}}$, and so $x_{3}^{*} \neq x_{4}^{*}$. Similarly, $x_{3}^{*} \neq x_{5}^{*}$. From $x_{4, n} \geqslant \tilde{u}_{1}$, we have $x_{4}^{*} \geqslant \tilde{u}_{1}=u_{1}+\delta_{0} \bar{e}_{0}$. Then, we can show that $x_{5}^{*} \neq x_{4}^{*}$. Thus, $x_{3}^{*}, x_{4}^{*}$ and $x_{5}^{*}$ are three distinct sign-changing solutions of (2.1).

Now we show the existence of the fourth sign-changing solution. For each $n \in \mathbb{N}$, let us define $\Omega_{i, n}(i=1,2,3,4)$ as above. For each $n \in \mathbb{N}$, we have

$$
\begin{gathered}
i\left(K_{n} G_{n}, \Omega_{1, n} \cap \Omega_{2, n},\left[u_{0}, v_{0}\right]\right)=1, \\
i\left(K_{n} G_{n}, \Omega_{3, n} \cap \Omega_{4, n},\left[u_{0}, v_{0}\right]\right)=1, \\
i\left(K_{n} G_{n}, \Omega_{4, n} \cap \Omega_{1, n},\left[u_{0}, v_{0}\right]\right)=1, \\
i\left(K_{n} G_{n},\left[u_{0}, v_{0}\right],\left[u_{0}, v_{0}\right]\right)=1 .
\end{gathered}
$$

Let

$$
\begin{aligned}
& \widetilde{D}_{1, n}=\Omega_{1, n} \backslash\left(C l_{\left[u_{0}, v_{0}\right]}\left(\Omega_{1, n} \cap \Omega_{4, n}\right) \cup\left(C l_{\left[u_{0}, v_{0}\right]}\left(\Omega_{1, n} \cap \Omega_{2, n}\right)\right),\right. \\
& \widetilde{D}_{2, n}=\Omega_{4, n} \backslash\left(C l_{\left[u_{0}, v_{0}\right]}\left(\Omega_{1, n} \cap \Omega_{4, n}\right) \cup\left(C l_{\left[u_{0}, v_{0}\right]}\left(\Omega_{4, n} \cap \Omega_{3, n}\right)\right) .\right.
\end{aligned}
$$

It follows from (2.31) and (2.44)-(2.46) that

$$
\begin{aligned}
& i\left(K_{n} G_{n}, \widetilde{D}_{1, n},\left[u_{0}, v_{0}\right]\right)=-1, \\
& i\left(K_{n} G_{n}, \widetilde{D}_{2, n},\left[u_{0}, v_{0}\right]\right)=-1 .
\end{aligned}
$$

By (2.27), (2.31) and (2.46)-(2.49), we have

$$
\begin{aligned}
& i\left(K_{n} G_{n},\left[u_{0}, v_{0}\right] \backslash\left(C l_{\left[u_{0}, v_{0}\right]} \widetilde{D}_{1, n} \cup C l_{\left[u_{0}, v_{0}\right]} \widetilde{D}_{2, n} \cup C l_{\left[u_{0}, v_{0}\right]}\left(\Omega_{1, n} \cap \Omega_{4, n}\right) \cup C l_{\left[u_{0}, v_{0}\right]} \Omega_{2, n}\right.\right. \\
& \left.\left.\quad \cup C l_{\left[u_{0}, v_{0}\right]} \Omega_{3, n} \cup \bar{U}\left(\theta, \bar{r}_{n}\right)\right),\left[u_{0}, v_{0}\right]\right)=1-(-1)-(-1)-1-1-1-( \pm 1)=\mp 1 .
\end{aligned}
$$

Therefore, for each $n \in \mathbb{N}, K_{n} G_{n}$ has a fixed point

$$
\begin{aligned}
x_{6, n} \in & {\left[u_{0}, v_{0}\right] \backslash\left(C l_{\left[u_{0}, v_{0}\right]} \widetilde{D}_{1, n} \cup C l_{\left[u_{0}, v_{0}\right]} \widetilde{D}_{2, n} \cup C l_{\left[u_{0}, v_{0}\right]}\left(\Omega_{1, n} \cap \Omega_{4, n}\right) \cup C l_{\left[u_{0}, v_{0}\right]} \Omega_{2, n}\right.} \\
& \left.\cup C l_{\left[u_{0}, v_{0}\right]} \Omega_{3, n} \cup \bar{U}\left(\theta, \bar{r}_{n}\right)\right) .
\end{aligned}
$$

Then, by the method of Theorem 2.1, we see that $\left\|x_{6, n}\right\|_{X} \geqslant \bar{r}_{0}$ for each $n \in \mathbb{N}$. Similar to the proof of Steps 5 and 6 in Theorem 2.1, we see that there exist a sub-sequence of $\left\{x_{6, n}\right\}_{n=1}^{\infty}$ (assume without loss of generality that the sub-sequence is $\left\{x_{6, n}\right\}_{n=1}^{\infty}$ itself) and $x_{6}^{*}$ such that $x_{6, n}^{*} \rightarrow x_{6}^{*}$ as $n \rightarrow \infty$ and $x_{6}^{*}$ is a sign-changing solution of (2.1). A 
similar way to that of showing (2.41)-(2.43) yields that $x_{6}^{*}$ is different from $x_{3}^{*}, x_{4}^{*}, x_{5}^{*}$. Thus, $x_{3}^{*}, \ldots, x_{6}^{*}$ are four sign-changing solutions of (2.1). The proof is complete.

REMARK 2.2. In [10] we obtained some existence results for sign-changing solutions of a three-point boundary value problem. The method to show the main results in [10] are different from that of this paper. We obtained the main results in [10] by using the modification functions technique and the Leray-Schauder degree method. But in this paper we obtained the main results by using the fixed point index method and the $\left(e_{1}, B\right)$-limit-increasing operator method.

THEOREM 2.3. Suppose that $\left(H_{1}\right)-\left(H_{4}\right)$ hold, $\beta_{0}<\frac{1}{\lambda_{1}}$. Then (2.1) has at least four sign-changing solutions. Moreover, (2.1) has at least two positive and two negative solutions.

Proof. The proof is similar to that of Theorem 2.2. For completeness, we will sketch the proof. Take $\left\{F_{n}\right\},\left\{M_{n}\right\}$ and $r_{0}$ such that (2.2)-(2.4) hold. Then $K_{n} G_{n}$ is Fréchet differentiable at $\theta, 1$ is not an eigenvalue of $\left(K_{n} G_{n}\right)^{\prime}(\theta)=\left(\beta_{0}+M_{n}\right) K_{n} B$ and $r\left(\left(K_{n} G_{n}\right)^{\prime}(\theta)\right)<1$ for each $n \in \mathbb{N}$. By Lemma 2.1, there exists $\tau_{n}>0$ such that for any $\tau \in\left(0, \tau_{n}\right]$

$$
-\tau h_{n}<K_{n} G_{n}\left(-\tau h_{n}\right), K_{n} G_{n}\left(\tau h_{n}\right)<\tau h_{n},
$$

where $h_{n}$ is the eigenfunction of $\left(K_{n} G_{n}\right)^{\prime}(\theta)$ corresponding to the eigenvalue $r\left(\left(K_{n} G_{n}\right)^{\prime}(\theta)\right)$.

For each $n \in \mathbb{N}$ and $i \in \mathbb{N}$, take $\varepsilon_{i} \in\left(0, \tau_{n}\right]$ small enough such that

$$
\begin{gathered}
u_{2, n}^{(i)} \nless v_{1}, u_{1} \nless v_{2, n}^{(i)}, \\
u_{0}<u_{2, n}^{(i)}<\theta<v_{2, n}^{(i)}<v_{0},
\end{gathered}
$$

and $\varepsilon_{i} \rightarrow 0$ as $i \rightarrow \infty$, where $u_{2, n}^{(i)}=-\varepsilon_{i} h_{n}, v_{2, n}^{(i)}=\varepsilon_{i} h_{n}$. Similar to the proof of Theorem 2.2, we may take $\delta_{0}>0$ small enough such that $L \widetilde{u}_{1} \prec_{1} F \widetilde{u}_{1}, F \widetilde{v}_{1} \prec_{1} L \widetilde{v}_{1}$ and $\tilde{u}_{1} \prec_{0} \widetilde{v}_{1}$, where $\tilde{u}_{1}=u_{1}+\delta_{0} \bar{e}_{0}$ and $\widetilde{v}_{1}=v_{1}-\delta_{0} \bar{e}_{0}$. Assume without loss of generality that (2.28)-(2.30) hold for all $n \in \mathbb{N}$. For each $n \in \mathbb{N}$ and $i \in \mathbb{N}$, let

$\Omega_{1, n}=\left\{x \in\left[u_{0}, v_{0}\right] \mid\right.$ there exists $\tau>0$ such that $\left.K_{n} G_{n} x \geqslant K_{n} G_{n} u_{1}+\tau \bar{e}_{n}\right\}$,

$\Omega_{2, n}^{(i)}=\left\{x \in\left[u_{0}, v_{0}\right] \mid\right.$ there exists $\tau>0$ such that $\left.K_{n} G_{n} x \geqslant K_{n} G_{n} u_{2, n}^{(i)}+\tau \bar{e}_{n}\right\}$,

$\Omega_{3, n}^{(i)}=\left\{x \in\left[u_{0}, v_{0}\right] \mid\right.$ there exists $\tau>0$ such that $\left.K_{n} G_{n} x \leqslant K_{n} G_{n} v_{2, n}^{(i)}-\tau \bar{e}_{n}\right\}$,

$\Omega_{4, n}=\left\{x \in\left[u_{0}, v_{0}\right] \mid\right.$ there exists $\tau>0$ such that $\left.K_{n} G_{n} x \leqslant K_{n} G_{n} v_{1}-\tau \bar{e}_{n}\right\}$,

$O_{1, n}=\left\{x \in\left[u_{0}, v_{0}\right] \mid\right.$ there exists $\tau>0$ such that $\left.K_{n} G_{n} x \geqslant K_{n} G_{n} \widetilde{u}_{1}+\tau \bar{e}_{n}\right\}$,

$O_{4, n}=\left\{x \in\left[u_{0}, v_{0}\right] \mid\right.$ there exists $\tau>0$ such that $\left.K_{n} G_{n} x \leqslant K_{n} G_{n} \widetilde{v}_{1}-\tau \bar{e}_{n}\right\}$,

$$
\begin{aligned}
& D_{1, n}^{(i)}=O_{1, n} \backslash\left(C l_{\left[u_{0}, v_{0}\right]}\left(O_{1, n} \cap \Omega_{4, n}\right) \cup C l_{\left[u_{0}, v_{0}\right]}\left(O_{1, n} \cap \Omega_{2, n}^{(i)}\right)\right), \\
& D_{2, n}^{(i)}=O_{4, n} \backslash\left(C l_{\left[u_{0}, v_{0}\right]}\left(O_{4, n} \cap \Omega_{1, n}\right) \cup C l_{\left[u_{0}, v_{0}\right]}\left(O_{4, n} \cap \Omega_{3, n}^{(i)}\right)\right), \\
& D_{3, n}^{(i)}=\Omega_{3, n}^{(i)} \backslash\left(C l_{\left[u_{0}, v_{0}\right]}\left(\Omega_{4, n} \cap \Omega_{3, n}^{(i)}\right) \cup C l_{\left[u_{0}, v_{0}\right]}\left(\Omega_{3, n} \cap \Omega_{2, n}^{(i)}\right)\right), \\
& D_{4, n}^{(i)}=\Omega_{1, n}^{(i)} \backslash\left(C l_{\left[u_{0}, v_{0}\right]}\left(\Omega_{1, n} \cap \Omega_{4, n}\right) \cup C l_{\left[u_{0}, v_{0}\right]}\left(\Omega_{1, n} \cap \Omega_{2, n}^{(i)}\right)\right) .
\end{aligned}
$$


Similar to the proof of Theorem 2.1, we can show that for each $n \in \mathbb{N}$ and some $i_{0} \in \mathbb{N}$,

$$
\begin{gathered}
i\left(K_{n} G_{n}, O_{1, n} \cap O_{4, n},\left[u_{0}, v_{0}\right]\right)=1, \\
i\left(K_{n} G_{n}, D_{j, n}^{\left(i_{0}\right)},\left[u_{0}, v_{0}\right]\right)=-1, j=1,2,3,4 .
\end{gathered}
$$

By (2.50) and (2.51), $K_{n} G_{n}$ has fixed points $x_{1, n} \in O_{1, n} \cap O_{4, n}, x_{2, n} \in D_{1, n}^{\left(i_{0}\right)}$ and $x_{3, n} \in$ $D_{2, n}^{\left(i_{0}\right)}$. Moreover,

$$
\begin{gathered}
i\left(K_{n} G_{n},\left[u_{0}, v_{0}\right],\left[u_{0}, v_{0}\right]\right)=1, \\
i\left(K_{n} G_{n}, \Omega_{2, n}^{\left(i_{0}\right)},\left[u_{0}, v_{0}\right]\right)=1, \\
i\left(K_{n} G_{n}, \Omega_{4, n},\left[u_{0}, v_{0}\right]\right)=1 .
\end{gathered}
$$

It follows from (2.51)-(2.54) that

$$
\begin{gathered}
i\left(K_{n} G_{n},\left[u_{0}, v_{0}\right] \backslash\left(C l_{\left[u_{0}, v_{0}\right]} \Omega_{2, n}^{\left(i_{0}\right)} \cup C l_{\left[u_{0}, v_{0}\right]} \Omega_{4, n} \cup C l_{\left[u_{0}, v_{0}\right]} D_{3, n}^{\left(i_{0}\right)}\right.\right. \\
\left.\left.\cup C l_{\left[u_{0}, v_{0}\right]} D_{4, n}^{\left(i_{0}\right)}\right),\left[u_{0}, v_{0}\right]\right)=1-1-1-(-1)-(-1)=1 .
\end{gathered}
$$

Therefore, $K_{n} G_{n}$ has a fixed point

$$
x_{4, n} \in\left[u_{0}, v_{0}\right] \backslash\left(C l_{\left[u_{0}, v_{0}\right]} \Omega_{2, n}^{\left(i_{0}\right)} \cup C l_{\left[u_{0}, v_{0}\right]} \Omega_{4, n} \cup C l_{\left[u_{0}, v_{0}\right]} D_{3, n}^{\left(i_{0}\right)} \cup C l_{\left[u_{0}, v_{0}\right]} D_{4, n}^{\left(i_{0}\right)}\right) .
$$

Since $\left[\theta, v_{0}\right] \subset \Omega_{2, n}^{\left(i_{0}\right)}$ and $\left[u_{0}, \theta\right] \subset \Omega_{3, n}^{\left(i_{0}\right)}$, we easily see that $x_{1, n}, x_{2, n}, x_{3, n}, x_{4, n} \in X \backslash(P \cup$ $(-P)$ ). By a similar way as that of Theorem 2.2 we can show that for each $n \in \mathbb{N}^{+}$there exists $\bar{r}_{n}>0$ such that $\bar{r}_{n}<\bar{r}_{0}<r_{0}$ and $K_{n} G_{n}$ has the unique solution $\theta$ in $U\left(\theta, \bar{r}_{n}\right)$. Then as the proof of Theorem 2.1, we can show that $\left\|x_{i, n}\right\|_{X} \geqslant \bar{r}_{0}$ for $i=1,2,3,4$ and $n \in \mathbb{N}$, and there exist sub-sequences of $\left\{x_{i, n}\right\}_{n=1}^{\infty}(i=1,2,3,4)$ (assume without loss of generality that the sub-sequences are $\left\{x_{i, n}\right\}_{n=1}^{\infty}(i=1,2,3,4)$ themselves $)$ and $x_{i}^{*}(i=1,2,3,4)$ such that $x_{i, n} \rightarrow x_{i}^{*}$ as $n \rightarrow \infty$ for each $i=1,2,3,4$. It is easy to see that $x_{1}^{*}, x_{2}^{*}, x_{3}^{*}$ and $x_{4}^{*}$ are four distinct sign-changing solutions of (2.1).

Next we shall show that (2.1) has at least two positive solutions. It is easy to see that for each $i, n \in \mathbb{N}$,

$$
i\left(K_{n} G_{n}, O_{1, n} \cap \Omega_{2, n}^{(i)},\left[u_{0}, v_{0}\right]\right)=1 .
$$

Then $K_{n} G_{n}$ has fixed point $x_{5, n}^{(i)} \in O_{1, n} \cap \Omega_{2, n}^{(i)}$. Similar to the above argument, we see that $\left\|x_{5, n}^{(i)}\right\|_{X} \geqslant \bar{r}_{0}$ for all $i, n \in \mathbb{N}$. Since $x_{5, n}^{(i)} \in O_{1, n} \cap \Omega_{2, n}^{(i)}$, we have

$$
x_{5, n}^{(i)}=K_{n} G_{n} x_{5, n}^{(i)} \geqslant K_{n} G_{n} u_{2, n}^{(i)}+\tau \bar{e}_{n} \geqslant K_{n} G_{n} u_{2, n}^{(i)}>u_{2, n}^{(i)} .
$$

A similar argument as given in Theorem 2.1 shows that there exist a sub-sequence of $\left\{x_{5, n}^{(i)}\right\}$ (assume without loss of generality that the sub-sequence is $\left\{x_{5, n}^{(i)}\right\}$ itself) and $x_{5}^{(i)}$ such that $x_{5, n}^{(i)} \rightarrow x_{5}^{(i)}$ as $n \rightarrow \infty$. Obviously, $x_{5}^{(i)}$ is a solution of $(2.1)$, and so $x_{5}^{(i)}=$ $K F x_{5}^{(i)}$. From $\left\{x_{5}^{(i)} \mid i=1,2, \ldots\right\} \subset\left[u_{0}, v_{0}\right]$, we see that $\left\{x_{5}^{(i)} \mid i=1,2, \ldots\right\}$ is bounded. Thus, $\left\{x_{5}^{(i)} \mid i=1,2, \ldots\right\}$ is a relatively compact set because $K$ is a completely continuous 
operator. Assume that $x_{5}^{(i)} \rightarrow x_{5}^{*}$ as $i \rightarrow \infty$, then $x_{5}^{*}=K F x_{5}^{*}$, that is $L x_{5}^{*}=F x_{5}^{*}$. This means that $x_{5}^{*}$ is a solution of (2.1). It follows from (2.56) that $x_{5}^{*} \geqslant \theta$. Since $\left\|x_{5, n}^{(i)}\right\|_{X} \geqslant$ $\bar{r}_{0}, x_{5}^{*}$ is not a zero solution of (2.1). Thus, $x_{5}^{*}$ is a positive solution of (2.1).

Also, we can show that for each $i \in \mathbb{N}$ and $n \in \mathbb{N}$ and some $j_{0} \in N$,

$$
i\left(K_{n} G_{n}, \Omega_{2, n}^{(i)} \backslash\left(C l_{\left[u_{0}, v_{0}\right]}\left(\Omega_{2, n}^{(i)} \cap \Omega_{3, n}^{\left(j_{0}\right)}\right) \cup C l_{\left[u_{0}, v_{0}\right]}\left(\Omega_{2, n}^{(i)} \cap \Omega_{1, n}\right)\right),\left[u_{0}, v_{0}\right]\right)=-1 .
$$

Thus, $K_{n} G_{n}$ has a fixed point $x_{6, n}^{(i)} \in \Omega_{2, n}^{(i)} \backslash\left(C l_{\left[u_{0}, v_{0}\right]}\left(\Omega_{2, n}^{(i)} \cap \Omega_{3, n}^{\left(j_{0}\right)}\right) \cup C l_{\left[u_{0}, v_{0}\right]}\left(\Omega_{2, n}^{(i)} \cap \Omega_{1, n}\right)\right)$. Similar to the above argument, we may assume that $x_{6, n}^{(i)} \rightarrow x_{6}^{(i)}$ as $n \rightarrow \infty$ and $x_{6}^{(i)} \rightarrow x_{6}^{*}$ as $i \rightarrow \infty$. Then $x_{6}^{*}$ is a positive solution of (2.1). It is easy to see that $x_{6}^{*} \neq x_{5}^{*}$. Therefore, $x_{5}^{*}$ and $x_{6}^{*}$ are two positive solutions of (2.1). Similarly, we can show that (2.1) has at least two negative solutions, $x_{7}^{*}$ and $x_{8}^{*}$. The proof is complete.

REMARK 2.3. In Theorems 2.2 and 2.3 we not only obtained multiplicity results for sign-changing solutions but also made a clear description of positions of these solutions of the non-linear operator equation (2.1). In order to show the main results, we have constructed some strict upper or lower solutions of (2.1). Some pairs of these strict upper and lower solutions are well ordered and others are not well ordered. Especially, some pairs of strict upper and lower solutions are parallel to each other. For other discussions concerning the parallel pairs of upper and lower solutions, the reader is refereed to $[\mathbf{1 0}, \mathbf{1 3}]$.

3. Applications of the abstract results in differential boundary value problems. In this section we will apply the main results of Section 2 to study the Sturm-Liouville differential boundary value problem

$$
\left\{\begin{array}{l}
-\left(p(t) u^{\prime}\right)^{\prime}-q(t) u=f(t, u(t)), \quad t \in I \\
R_{0}(u):=a u(0)-b u^{\prime}(0)=0 \\
R_{1}(u):=c u(1)+d u^{\prime}(1)=0
\end{array}\right.
$$

where $I=[0,1], p(t) \in C^{1}(I), q(t) \leqslant 0, p(t)>0(\forall t \in I), a, b, c, d \geqslant 0, a^{2}+b^{2} \neq 0, c^{2}+$ $d^{2} \neq 0$.

Let $X=C(I)$ denote the Banach space of all continuous functions on $I$ with the maximum norm $\|\cdot\|_{X}$, and $Z=X \times R^{2}$. For each $\tilde{x}=(x(t), l, m) \in Z$, let

$$
\|\tilde{x}\|_{Z}=\|x(t)\|_{X}+|l|+|m| .
$$

Then $\left(Z,\|\cdot\|_{Z}\right)$ is the real Banach space. Let $P=\{x=x(t) \in X \mid x(t) \geqslant 0, t \in I\}$ and $P_{1}=\{\tilde{x}=(x(t), l, m) \in Z \mid x(t) \in P, l \geqslant 0, m \geqslant 0\}$. Then $P$ and $P_{1}$ are normal cones of $X$ and $Z$, respectively.

Now let us introduce the following conditions to be used.

$\left(\mathrm{A}_{1}\right) f \in C\left(I \times R^{1}, R^{1}\right), f(t, 0)=0$, and $f(t, x)$ is locally continuous differentiable with $x$ at $x=0$.

$\left(\mathrm{A}_{2}\right)$ There exists $\beta_{0}>0$ such that $\lim _{x \rightarrow 0} \frac{f(t, x)}{x}=\beta_{0}$ uniformly with $t \in I$.

$\left(\mathrm{A}_{3}\right)$ There exist $u_{0}(t), v_{0}(t) \in X, u_{0}(t)<0<v_{0}(t)$ for all $t \in I, u_{0}, v_{0}$ are strict lower and upper solutions of (3.1).

(A $\left.\mathrm{A}_{4}\right)$ There exist $u_{1}, v_{1} \in X$ such that $u_{1}$ and $v_{1}$ are sign-changing on $I$ and $u_{0}(t)<$ $u_{1}(t)<v_{1}(t)<v_{0}(t)$ for all $t \in I, u_{1}, v_{1}$ are strict lower and upper solutions of (3.1). 

problem:

Let $\left\{\lambda_{n}\right\}$ denote the decreasing sequence of the positive eigenvalues of the linear

$$
\left\{\begin{array}{l}
-\left(p(t) u^{\prime}\right)^{\prime}-q(t) u=\frac{1}{\lambda_{n}} u, t \in(0,1) \\
R_{0}(u)=R_{1}(u)=0
\end{array}\right.
$$

The following Lemmas 3.1-3.3 can be found in Section 3 of [13].

LEMMA 3.1. For each $M \geqslant 0$, let $\varphi_{M}(t)$ and $\psi_{M}(t)$ satisfy

$$
\begin{aligned}
& \left\{\begin{array}{l}
\left(p(t) \varphi_{M}^{\prime}(t)\right)^{\prime}+(q(t)-M) \varphi_{M}(t)=0, \quad t \in I, \\
\varphi_{M}(1)=d, \varphi_{M}^{\prime}(1)=-c,
\end{array}\right. \\
& \left\{\begin{array}{l}
\left(p(t) \psi_{M}^{\prime}(t)\right)^{\prime}+(q(t)-M) \psi_{M}(t)=0, \quad t \in I, \\
\psi_{M}(0)=b, \psi_{M}^{\prime}(0)=a,
\end{array}\right.
\end{aligned}
$$

respectively. Then we have

(i) $\varphi_{M}(t)$ is non-increasing in $[0,1)$ with $\varphi_{M}(t)>0(t \in[0,1))$.

(ii) $\psi_{M}(t)$ is non-decreasing in $(0,1]$ with $\psi_{M}(t)>0(t \in(0,1])$.

(iii) $\varphi_{M}$ and $\psi_{M}$ are linearly independent.

(iv) $p(t)\left(\varphi_{M}(t) \psi_{M}^{\prime}(t)-\varphi_{M}^{\prime}(t) \psi_{M}(t)\right)=\omega$, where $\omega$ is a positive constant.

(v) $R_{0}\left(\varphi_{M}\right) \neq 0, R_{1}\left(\varphi_{M}\right)=0$.

(vi) $R_{0}\left(\psi_{M}\right)=0, R_{1}\left(\psi_{M}\right) \neq 0$.

Lemma 3.2. Let $g \in C(I), m, l \in R^{1}$. Then $u \in C^{2}(I)$ is a solution to the following boundary value problem

$$
\left\{\begin{array}{l}
-\left(p(t) u^{\prime}\right)^{\prime}-(q(t)-M) u=g(t), \quad t \in I, \\
R_{0}(u)=l, R_{1}(u)=m
\end{array}\right.
$$

if and only if

$$
u(t)=\frac{\varphi_{M}(t)}{R_{0}\left(\varphi_{M}\right)} l+\frac{\psi_{M}(t)}{R_{1}\left(\psi_{M}\right)} m+\int_{0}^{1} G(t, s) g(s) d s, \quad t \in I,
$$

where

$$
G_{M}(t, s)=\frac{1}{\omega} \begin{cases}\varphi_{M}(t) \psi_{M}(s), & s \leqslant t \\ \varphi_{M}(s) \psi_{M}(t), & s>t\end{cases}
$$

LeMmA 3.3. For any $M \geqslant 0, x \in P, l, m \geqslant 0$, let

$$
y(t)=\frac{\varphi_{M}(t)}{R_{0}\left(\varphi_{M}\right)} l+\frac{\psi_{M}(t)}{R_{1}\left(\psi_{M}\right)} m+\int_{0}^{1} G_{M}(t, s) x(s) d s, \quad t \in I .
$$

Then

$$
y(t) \geqslant\|y\|_{X} \bar{e}_{M}(t), \quad t \in I,
$$

where $\bar{e}_{M}(t)=\frac{1}{\gamma_{M}^{2}} \varphi_{M}(t) \psi_{M}(t), \gamma_{M}=\max \left\{\left\|\varphi_{M}\right\|_{X},\left\|\psi_{M}\right\|_{X}\right\}$. 
THEOREM 3.1. Suppose $\left(A_{1}\right)-\left(A_{3}\right)$ hold, and $\beta_{0} \in\left(\frac{1}{\lambda_{2 n_{0}}}, \frac{1}{\lambda_{2 n_{0}+1}}\right)$, where $n_{0}$ is a natural number. Then (3.1) has at least one sign-changing solution. Moreover, (3.1) has at least one positive and one negative solution.

Proof. Let us define the linear operators $L$ : dom $\mathrm{L}=C^{2}(I) \subset X \mapsto Z, B: X \mapsto Z$ and the non-linear operator $F: X \mapsto Z$ by

$$
\begin{gathered}
L x=\left(-\left(p(t) x^{\prime}(t)\right)^{\prime}-q(t) x(t), a x(0)-b x^{\prime}(0), c x(1)+d x^{\prime}(1)\right), \\
B x=(x(t), 0,0), F x=(f(t, x(t)), 0,0),
\end{gathered}
$$

respectively. Then, we need to consider the operator equation

$$
L u=F u, \quad u \in \operatorname{dom} L .
$$

For each $M \geqslant 0$, by Lemma 3.1-3.3 and a method in [13] we can easily show that $(L+M B)^{-1}$ exists, $(L+M B)^{-1}$ is $\bar{e}_{M}$-positive and $\bar{e}_{M}$-continuous (the details of the proof, one can find in Section 3 of [13]). By the well-known Sturm-Liouville theory of linear boundary value problems, we easily see that the algebraic multiplicity of each positive eigenvalue of $K_{M} B$ is 1 . Thus, $\left(\mathrm{H}_{3}\right)$ holds.

Since $f(t, x)$ is locally continuous differentiable with $x$ at $x=0$, there exist $r_{0}>0$ and $\tau_{0}>0$ such that

$$
f\left(t, x_{2}\right)-f\left(t, x_{1}\right) \geqslant-\tau_{0}\left(x_{2}-x_{1}\right), \quad \forall x_{2}, x_{1} \in\left[-r_{0}, r_{0}\right], x_{2}>x_{1}, \quad t \in I .
$$

Let $R_{0}>r_{0}$. Now since $f: I \times\left[r_{0}, R_{0}\right] \mapsto R^{1}$ is continuous, for any $n \in \mathbb{N}$, there exists $\widetilde{g}_{1}^{(n)}: I \times\left[r_{0}, R_{0}\right] \mapsto R^{1}$ infinitely differentiable such that

$$
\left|f(t, x)-g_{1}^{(n)}(t, x)\right|<\frac{1}{2 n}, \forall(t, x) \in I \times\left[r_{0}, R_{0}\right] .
$$

Let

$$
g_{1, n}(t, x)=\widetilde{g}_{1}^{(n)}(t, x)-\left(\widetilde{g}_{1}^{(n)}\left(t, r_{0}\right)-f\left(t, r_{0}\right)\right), \quad \forall(t, x) \in I \times\left[r_{0}, R_{0}\right]
$$

Then, $g_{1, n}$ is infinitely differentiable with $x$, and $g_{1, n}\left(t, r_{0}\right)=f\left(t, r_{0}\right)(\forall t \in I)$. For any $n \in \mathbb{N}$, we have

$$
\begin{aligned}
& \left|f(t, x)-g_{1, n}(t, x)\right| \leqslant\left|f(t, x)-\widetilde{g}_{1}^{(n)}(t, x)\right|+\left|\widetilde{g}_{1}^{(n)}\left(t, r_{0}\right)-f\left(t, r_{0}\right)\right|<\frac{1}{n}, \quad \forall(t, x) \in I \\
& \quad \times\left[r_{0}, R_{0}\right] .
\end{aligned}
$$

Since $g_{1, n}$ is infinitely differentiable with $x$, there exists $\tau_{1, n}>0$ such that

$$
g_{1, n}\left(t, x_{2}\right)-g_{1, n}\left(t, x_{1}\right)>-\tau_{1, n}\left(x_{2}-x_{1}\right), \quad \forall\left(t, x_{1}\right),\left(t, x_{2}\right) \in I \times\left[r_{0}, R_{0}\right], x_{2}>x_{1} .
$$

Similarly, there exists $g_{2, n}(t, x)$, which is continuous on $I \times\left[-R_{0},-r_{0}\right]$ and infinitely differentiable such that $g_{2, n}\left(t,-r_{0}\right)=f\left(t,-r_{0}\right)(\forall t \in I)$, and

$$
\left|f(t, x)-g_{2, n}(t, x)\right|<\frac{1}{n}, \quad \forall(t, x) \in I \times\left[-R_{0},-r_{0}\right] .
$$

Also, there exists $\tau_{2, n}>0$ such that

$$
g_{2, n}\left(t, x_{2}\right)-g_{2, n}\left(t, x_{1}\right)>-\tau_{2, n}\left(x_{2}-x_{1}\right), \quad \forall\left(t, x_{2}\right),\left(t, x_{1}\right) \in I \times\left[-R_{0},-r_{0}\right], x_{2}>x_{1} .
$$


For each $n \in \mathbb{N}$, let $f_{n}: I \times\left[-R_{0}, R_{0}\right] \mapsto R^{1}$ be defined by

$$
f_{n}(t, x)= \begin{cases}g_{1, n}(t, x), & (t, x) \in I \times\left[r_{0}, R_{0}\right] ; \\ f(t, x), & (t, x) \in I \times\left[-r_{0}, r_{0}\right] ; \\ g_{2, n}(t, x), & (t, x) \in I \times\left[-R_{0},-r_{0}\right] .\end{cases}
$$

Then, $f_{n} \in C\left(I \times\left[-R_{0}, R_{0}\right]\right)$, and for each $n \in \mathbb{N}$,

$$
\left|f(t, x)-f_{n}(t, x)\right|<\frac{1}{n}, \forall(t, x) \in I \times\left[-R_{0}, R_{0}\right] .
$$

Let $M_{n}=\tau_{1, n}+\tau_{2, n}+\tau_{0}$. Then we have

$$
f_{n}\left(t, x_{2}\right)-f_{n}\left(t, x_{1}\right)>-M_{n}\left(x_{2}-x_{1}\right), \quad \forall\left(t, x_{2}\right),\left(t, x_{1}\right) \in I \times\left[-R_{0}, R_{0}\right], x_{2}>x_{1} .
$$

Define $F_{n}: X \mapsto Z$ by

$$
F_{n} x=\left(f_{n}(t, x(t)), 0,0\right)
$$

Then we have

$$
-\frac{1}{n} e_{1}<F_{n} x-F x<\frac{1}{n} e_{1},
$$

where $\bar{x}_{0}(t) \equiv 1(\forall t \in I)$ and $e_{1}=\left(\bar{x}_{0}(t), 0,0\right)$. For each $x \in U\left(\theta, r_{0}\right)$ we have

$$
F_{n} x=F x, n=1,2, \ldots
$$

Therefore, $F$ is $\left(e_{1}, B\right)$-limit increasing on any bounded set of $X$ and is locally increasing at $\theta$. It follows from $\left(\mathrm{A}_{2}\right)$ that $F$ is Fréchet differentiable at $\theta$ and $F^{\prime}(\theta)=\beta_{0} B$. We see from the continuity of $f$ that $F$ is $e_{1}$-continuous. This means that $\left(\mathrm{H}_{2}\right)$ holds. From $\left(\mathrm{A}_{3}\right)$ we see that $u_{0}$ and $v_{0}$ are strict lower and upper solutions of (3.1), respectively. This means that $\left(\mathrm{H}_{1}\right)$ holds. Thus, all conditions of Theorem 2.1 are satisfied. Now the conclusion of Theorem 3.1 follows from Theorem 2.1. The proof is complete.

By Theorems 2.2 and 2.3 we can show the following Theorems 3.2. and 3.3.

THEOREM 3.2. Suppose that $\left(A_{1}\right)-\left(A_{4}\right)$ hold, $\beta_{0}>\frac{1}{\lambda_{1}}, \beta_{0} \neq \frac{1}{\lambda_{n}}$ for all $n \geqslant 2$. Then the boundary value problem (3.1) has at least four sign-changing solutions. Moreover, the boundary value problem (3.1) has at least one positive and one negative solution.

THEOREM 3.3. Suppose that $\left(A_{1}\right)-\left(A_{4}\right)$ hold, $\beta_{0}<\frac{1}{\lambda_{1}}$. Then the boundary value problem (3.1) has at least four sign-changing solutions. Moreover, the boundary value problem (3.1) has at least two positive and two negative solutions.

REMARK 3.1. In Theorems 2.2 and 2.3 we have employed a condition of a pair of well-ordered strict lower and upper solutions that are sign-changing. As pointed out in [10], it is very difficult to construct a pair of well-ordered strict lower and upper solutions. However, a concrete numeral example shows that such a pair of well-ordered strict lower and upper solutions does exist, see [10].

REMARK 3.2. In Theorems 2.1-2.3, by combining the fixed point index method and the concept of $\left(e_{1}, B\right)$-limit-increasing operator we have obtained some multiplicity results for sign-changing solutions. Obviously, if we combine the critical point theory 
method with the concept of $\left(e_{1}, B\right)$-limit-increasing operator, we may obtain some interesting results for sign-changing critical point of non-linear boundary value problems.

\section{REFERENCES}

1. T. Bartsch and Z. Q. Wang, Sign changing solutions of nonlinear Schrodinger equations, Topo. Meth. Nonlinear Anal. 13 (1999), 191-198.

2. E. N. Dancer and Y. Du, Existence of changing sign solutions for some semilinear problems with jumping nonlinearities at zero, Proc. R. Soc. Edinb. 124 A (1994), 1165-1176.

3. E. N. Dancer and Y. Du, On sign-changing solutions of certain semilinear elliptic problems, Appl. Anal. 56 (1995), 193-206.

4. T. Bartsch, Critical point theory on partially ordered Hilbert spaces, J. Funct. Anal. 186 (2001), 117-152.

5. T. Bartsch and Z. Q. Wang, On the existence of sign-changing solutions for semilinear Dirichlet problems, Topo. Meth. Nonlinear Anal. 7 (1996), 115-131.

6. Z. Liu, Localized critical points in Banach spaces and sign changing solutions of nonlinear p-Laplacian equations. Topological methods, variational methods (World Scientific Press, New Jersery, 2002).

7. T. Bartsch, K. C. Chang and Z. Q. Wang, On the Morse indices of sign changing solutions of nonlinear elliptic problems, Math. Z. 233 (2000), 655-677.

8. X. Xian and S. Jingxian, On sign-changing solution for some three-point boundary value problems, Nonlinear Anal. 59 (2004), 491-505.

9. X. Xian, Multiple sign-changing solutions for some $m$-point boundary value problems, Electron. J. Differ. Equ. 2004 (89) (2004), 1-14.

10. X. Xian and D. O'Regan, Multiplicity of sign-changing solutions for some four-point boundary value problem, Nonlinear Anal. 69 (2008), 434-447.

11. X. Xian, S. Jingxian and D. O'Regan, Nodal solutions for $m$-point boundary value problems using bifurcation methods, Nonlinear Anal. 68 (2008), 3034-3046.

12. Z. Wenming and M. Schechter, Critical points theory and its applications (Springer Verlag, New York, NY, 2006).

13. X. Xian and S. Jingxian, Solutions for an operator equation under the conditions of pairs of paralleled lower and upper solutions, Nonlinear Anal. 69 (2008), 2251-2266.

14. H. Amann, On the number of solutions of nonlinear equations in ordered Banach spaces, J. Funct. Anal. 11 (1972), 346-384.

15. F. Li. Solutions of nonlinear operator equations and applications. PhD Thesis (Shandong University, 1996).

16. H. Amann, Fixed point equations and nonlinear eigenvalue problems in ordered Banach spaces, SIAM Rev. 18 (1976), 620-709.

17. K. Deimling, Nonlinear functional analysis (Springer Verlag, New York, NY, 1985).

18. S. Jingxian and $X$. Xian, Three solution theorems for nonlinear operator equations and applications, J. Syst. Sci. Complex 18 (1) (2005), 119-125.

19. G. Dajun, Nonlinear functional analysis and applications (Beijing Sci. \& Tec. Press, Beijing, China, 1994).

20. G. Dajun and V. Lakshmikantham, Nonlinear problems in abstract cones (Academic Press, New York, NY, 1988).

21. S. Carla and D. Motreanu, Constant-sign and sign-changing solutions for nonlinear eigenvalue problems, Nonlinear Anal. 68 (2008) 2668-2676.

22. Y. Li and Z. L. Liu, Multiple and sign-changing solutions of an elliptic eigenvalue problem with constraint, Sci. China (Series A), 44(1) (2001), 48-57.

23. Z.-Q. Wang, Sign-changing solutions for a class of nonlinear elliptic problems, in Nonlinear analysis (Chang K.-C. and Long Y., Editors), Nankai Series in Pure and Applied Math. 6 (2000), 370-383.

24. Z. Zhang and $\mathrm{S}$. Li, On sign-changing and multiple solutions of the p-Laplacian, J. Funct. Anal. 197 (2003), 447-468.

25. Z. Zhang and K. Perera, Sign-changing solutions of Kirchhof-type problems via invariant sets of descent flow, J. Math. Anal. Appl. 317 (2006), 456-463. 
26. L. Shujie and W. Zhi-Qiang, Ljusternik-Schnirelman theory in partially ordered Hilbert spaces, Trans. Amer. Math. Soc. 354(8) (2002), 3207-3227.

27. L. Shujie and W. Zhi-Qiang, Mountain pass theorem in order intervals and multiple solutions for semilinear elliptic Dirichlet problems, J. Anal. Math. 81 (2000), 373-396.

28. Z. Wenming, Sign-changing critical point theory (Springer-Verlag, New York, NY, 2008).

29. P. H. Rabinowitz, Some global results for nonlinear eigenvalues, J. Funct. Anal. 7 (1971), 487-513.

30. P. H. Rabinowitz, On bifurcation from infinity, J. Differ. Equ. 14 (1973), 462-475. 